\title{
EL MODELO DE ADAPTACIÓN DE ROY EN EL CONTEXTO DE LOS MODELOS DE ENFERMERÍA, CON EJEMPLOS DE APLICACIÓN Y DIFICULTADES
}

\author{
C. Roy Sr. Callista Roy, PhD, RN, FAAN \\ Professor and Nurse Theorist. Boston College School of Nursing
}

\section{RESUMEN}

En este trabajo hablaremos sobre los modelos de enfermería en general. En segundo lugar, describiré el MAR específicamente, incluyendo las premisas filosóficas y científicas, así como los conceptos clave. En tercer lugar, les daré ejemplos de aplicación del modelo en la práctica, y concluiré con la discusión de algunas dificultades encontradas al utilizar el método en la educación de las enfermeras.

Al desarrollar los modelos para la práctica enfermera en los Estados Unidos en los 70 y 80, se comprobó que a través de los diferentes modelos, podíamos encontrar elementos básicos comunes. Los modelos incluyen conceptos claros de la per- sona. La comprensión del ser humano está basada en nuestra filosofía al igual que en nuestra visión científica de las personas y su lugar en el mundo que nos rodea. Además, los modelos de enfermería describen el mundo en el que las personas interactúan, denominándolo medio ambiente. Los otros dos conceptos principales son las nociones de salud y la idea de enfermería en sí misma. Entonces, los modelos de enfermería son descripciones de la práctica enfermera que se expresan utilizándolos conceptos esenciales de persona, medio ambiente, salud y enfermería.

Palabras clave: teoria enfermera, modelos de enfermería.

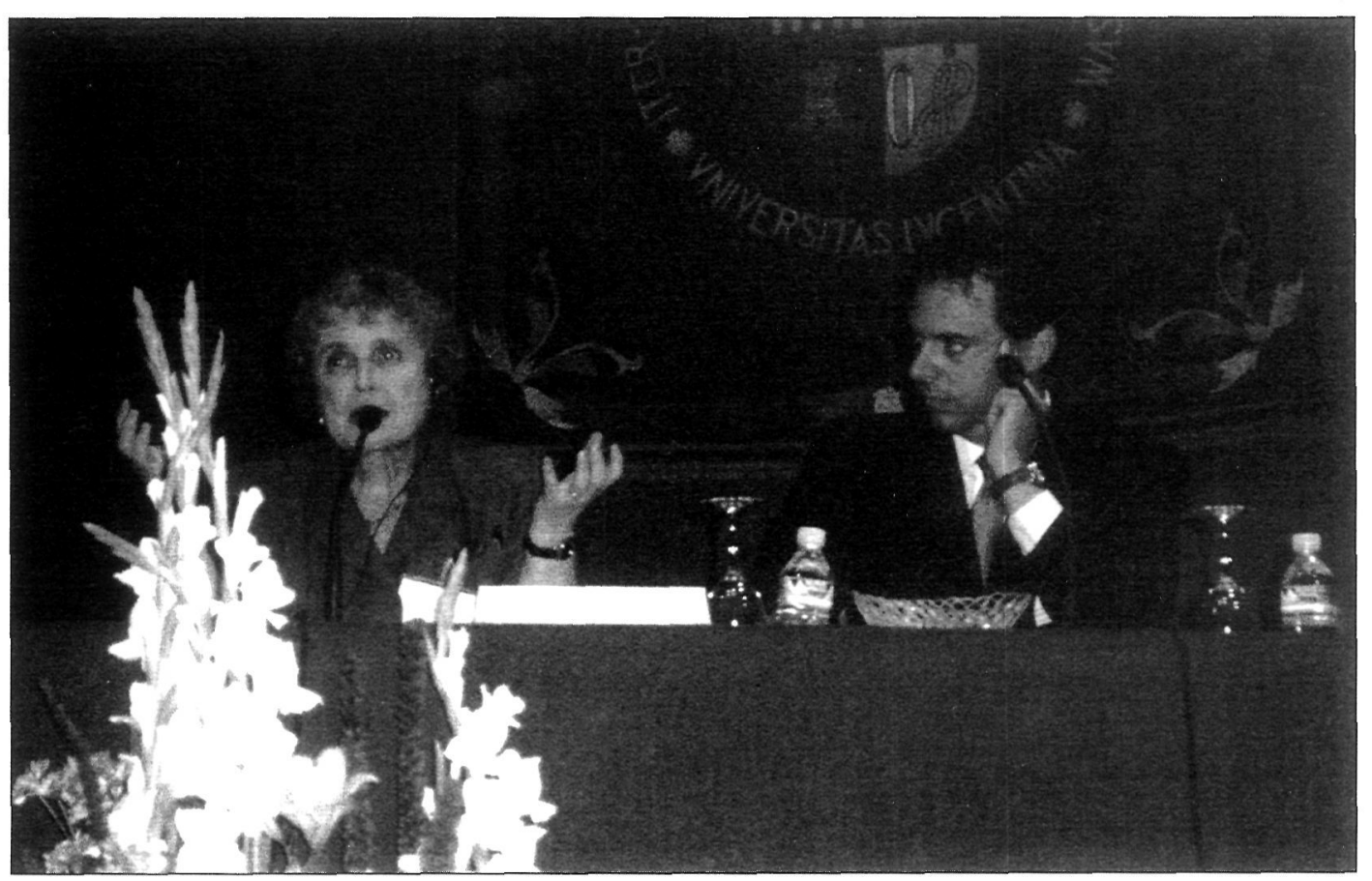




\section{SUMMARY}

In this work we will talk about nursing models in general. Secondly, I will describe the RAM specifically, including the philosophical and scientific assumptions as well as the key concepts. Thirdly, I will provide examples of applications of the model in practice and conclude with a discussion of some of the difficulties encountered when the model is used in practice and in nursing education. As models for nursing practice began to be developed in the United States in the 1970s and the 1980s, it was noted that looking across different models, we could find the same basic elements. Models include a clear concept of the person. Understanding the human person is based on our philosophy as well as our scientific view of persons and their place in the world around. In addition, nursing models in describing the world that people interact with are describing what we call environment. The other two major concepts are the notion of health and the idea of nursing itself. Nursing models then are depictions of nursing practice that are expressed using the essential concepts of person, environment, health, and nursing.

Keywords: Nursing theory, nursing models.

\section{Modelos de Enfermería.}

Un modelo es simplemente una representación de la realidad. Cuando se construye una nueva sección de un hospital, es habitual que se coloque una maqueta en la entrada del antiguo hospital, que suele ser de plástico o madera representando el edificio proyectado. Por ejemplo, puede representar como el nuevo edificio de consultas externas se conectará con el parking y con los otros edificios ya construidos. La maqueta representa como quedará la nueva construcción, y como se relacionará con las otras. La enfermería ha encontrado útil desarrollar modelos que represen- ten la realidad de la enfermería, para ver las distintas partes, y como se relacionan entre ellas. Estos modelos se construyen de conceptos y figuras que representan las partes de la enfermería. Otro ejemplo de modelo puede ser el artista pintando un árbol. Todos hemos visto árboles preciosos pintados por Van Gogh. También ha pintado flores en los árboles al hacerlo de cerca. Un modelo, entonces, nos proporciona una vista general y pormenorizada de las partes que representan nuestras creencias sobre la enfermería.

Es importante reconocer que los modelos de enfermería son representaciones de la realidad de la práctica enfermera. Lo que ocurre entre pacientes y enfermeras, y cómo estas contribuyen al cuidado y a la mejora de la salud es el objeto principal de los modelos de enfermería. Al desarrollar los modelos para la práctica enfermera en los Estados Unidos en los 70 y 80 , se comprobó que a través de los diferentes modelos, podíamos encontrar elementos básicos comunes. Los modelos incluyen conceptos claros de la persona. La comprensión del ser humano está basada en nuestra filosofía al igual que en nuestra visión científica de las personas y su lugar en el mundo que nos rodea. Además, los modelos de enfermería describen el mundo en el que las personas interactúan, denominándolo medio ambiente. Los otros dos conceptos principales son las nociones de salud y la idea de enfermería en sí misma. Entonces, los modelos de enfermería son descripciones de la práctica enfermera que se expresan utilizándo los conceptos esenciales de persona, medio ambiente, salud y enfermería.

\section{El Modelo de Adaptación de Roy (MAR).}

Como modelo de enfermería, el MAR tiene unas premisas filosóficas y científicas que relacionan específicamente a la persona y al mundo en el cual interactúan. Los avances científicos del siglo $\mathrm{XX}$, particularmente la teoría de los quantos y las exploraciones del universo, han abierto el camino 
para nuevas visiones de la persona y de su medio ambiente en el nuevo milenio. Basándome en estos avances, quisiera compartir con ustedes mi más reciente trabajo en las premisas de modelo de Roy para el siglo XXI.

Las premisas científicas para el siglo XXI están basadas en las de los sistemas previos y en las relacionadas con la adaptación. Aún así, se han añadido ideas para la comprensión del lugar de las personas en el universo. Estas premisas son:

1. Los sistemas de materia y energía progresan a niveles superiores de autoorganización compleja.

2. La consciencia y el significado son constitutivos de la integración de la persona y el medio ambiente.

3. Reconocimiento de sí mismo y del medio está enraizado en el pensamiento y en los sentidos

4. Las decisiones humanas son básicas para la integración de los procesos creativos.

5. El pensamiento y los sentimientos mediatizan las acciones humanas.

6. Los sistemas de inter-relación incluyen aceptación, protección y promoción de las relaciones de inter-dependencia.

7. Las personas y la tierra tienen caminos comunes y relaciones integrales.

8. Las transformaciones de las personas y del medio ambiente son creadas por la conciencia humana.

9. La integración de los significados de ser humano y medio ambiente tiene como resultado la adaptación.

Las dos premisas básicas que usé en 1988 fueron: 1. Humanismo, que es, el movimiento filosófico y psicológico que reconoce a la persona y a la dimensión subjetiva de la experiencia humana como valor central de conocimiento.

2. Verificabilidad, que es un principio de la naturaleza humana que afirma un objetivo común de la existencia humana.

A partir de aquí y de más experiencias recientes y comunidades escolares de fundaciones religiosas, empecé a desarrollar las premisas filosóficas para el siglo XXI. Estas incluyen:

1. Las personas tienen relaciones con el mundo y con una figura-Dios

2. El significado humano tiene raíces en un punto de convergencia omega del universo.

3. Dios está íntimamente revelado en la diversidad de la creación y es el destino común de la creación. 4. Las personas usan las habilidades creativas humanas de reconocer, descubrir y tener fe.

5. Las personas son importantes para los procesos de derivación, sostén y transformación del universo.

Me gustaría resumir este apartado de premisas, haciendo notar simplemente que a este desarrollo le he llamado unidad cósmica. Esto se refiere a la compleja autoorganización de las personas y el medio ambiente, y en segundo lugar, el significativo destino de convergencia de las personas y el medio ambiento, en lo que he denominado Dioscreador.

Al describir los conceptos clave del modelo, lo primero es describir a la persona o grupo como un sistema adaptativo con procesos de convivencia. El sistema adaptativo se describe como un todo compuesto por partes. Este funciona como una unidad con el mismo objetivo. Finalmente, los sistemas humanos incluyen a la gente individualmente o como grupo. Estos pueden ser familias, organizaciones, comunidades o la sociedad como un todo. Dos elementos clave para la comprensión de la persona como un sistema adaptativo son los procesos adaptativos y los modos de adaptación. Los procesos adaptativos incluyen la actividad de los subsistemas para convivir en el nivel individual o grupal. Los modos de adaptación son vistos como vías de manifestación de los procesos adaptativos. Los procesos centrales de adaptación son principalmente descritos como el subsistema regulador y el subsistema cognitivo. El subsistema regulador incluye todos los canales neuroquímicos 
y endocrinos por los que la persona reacciona al medio mediante respuesta casi automáticas e inconscientes. En segundo lugar, el subsistema cognitivo procesa la información perceptual, aprendizaje, juicios y emociones, de tal manera que los estímulos externos e internos son procesados para que la persona pueda realizar una respuesta.

La historia que suelo contar para ilustrar estos dos conceptos abstractos es la de una madre de un niño pequeño enfrente de su casa. Imagínense al niño corriendo hacia la calle cuando viene un coche. La madre corre y empuja al niño fuera de la calle. En ese caso, su sistema regulador actuó para darle toda la adrenalina y los neurotransmisores que necesitaba para actuar rápidamente. La información no llega al córtex cerebral. Más bien, viaja por la médula espinal y le permite actuar rápidamente. En segundo lugar, una vez que el niño se encuentra a salvo en sus brazos, empieza a pensar sobre la situación, entonces se de cuenta la ansiedad y el miedo que siente. Hace un nuevo juicio y concluye que el niño es muy activo y que necesitará vallar su jardín. Estos procesos se dan juntos para permitir a la persona adaptarse a los cambios del medio ambiente, tanto de inmediato como a largo plazo.

Los procesos internos paralelos de un grupo son el subsistema estabilizador y el subsistema innovador, estos serán discutidos más tarde en un ejemplo.

En 500 muestras sobre el comportamiento de los pacientes se identificaron 4 modos de adaptación. El primero ha sido redefinido como el modo físico y psicológico. Las características de este modo incluyen las formas en que los humanos interactúan como seres físicos. Esto incluye nueve componentes para el individuo con el sustrato de la necesidad de mantener la integridad fisiológica. Para el individuo, el modo fisiológico incluye 5 necesidades básicas: oxigenación, nutrición, elimi- nación, actividad y descanso, y protección. Además, el modo fisiológico incluye componentes que se basan en el subsistema regulador, que son los sentidos, fluidos y electrolitos, las funciones neurológicas, y endocrinas. Para los grupos, el modo físico comprende las manifestaciones adaptativas relacionadas con los recursos para las operaciones básicas, como los participantes, lacilidades físicas, recursos fiscales.

El siguiente modo adaptativo se refiere al autoconcepto. Esto es lo que es uno para sí mismo en algún momento. La mujer de la figura está en un bote en el Titicaca, en Sudamérica. El autoconcepto o identidad de grupo se describe como: la necesidad subyacente del individuo, es la integridad física, o la necesidad de saber quién es uno, para ser o existir con un sentido de unidad. La necesidad subyacente para el grupo es la identidad/ integridad. Los componentes del modo del autoconcepto para el individuo incluyen el yo físico, el cual se manifiesta por las sensaciones corporales y la propia imagen. En segundo lugar, el yo personal incluye la autoconsistencia, el yo ideal, y el yo moral, ético y espiritual. Los conceptos del modo de identidad de grupo incluye las relaciones interpersonales, autopercepción del grupo, el medio social y cultural. La figura muestra el modo de identidad de grupo con sus sub-áreas y componentes. La identidad de grupo se nutre de compartir las relaciones, valores y objetivos. Así, esta visión compartida reposa sobre la propia imagen del grupo y la co-responsabilidad en el cumplimiento de los objetivos. Esto ocurre en el interior del contexto general del medio social y cultural.

Además, de entre la larga muestra de comportamiento de pacientes, identificamos necesidades relacionadas con lo que denominamos el modo de función de rol. En esta fotografía, vemos a una mujer con muchos roles, madre, amiga, compradores y vendedores en un mercado al aire abierto. La necesidad subyacente del modo de función de 
rol para el individuo es la integración social; la necesidad de saber quién es uno en relación con los otros para así poder actuar. Para cualquier grupo la necesidad subyacente del modo de función de rol es la clarificación del rol. Es útil determinar los roles para describir el rol una determinada persona en el conjunto. Este conjunto de roles incluye los primarios, que están basados en el estado evolutivo de cada uno, por ejemplo una mujer de 27 años. Esta persona puede tener muchos roles secundarios que estén relacionados con el primario, como el de esposa, madre, maestra. Finalmente, los terciarios incluyen aquelios que son menos centrales para la persona. Por ejemplo, consultor de moda, cocinero tres tardes a la semana, aficionado al baloncesto, estudiante universitario a tiempo parcial, miembro de una asociación, y entrenador de fútbol.

Actualizando la literatura sobre el modo de función de rol, hemos añadido términos relacionados con los conjuntos de roles siendo los roles del individuo de la persona y los roles añadidos, siendo estos usados por muchas personas al mismo tiempo. Además, nuestra comprensión del modo de la función de rol la aproximación estructural. Esto describe los comportamiento instrumentales, es decir, comportamientos usados para conseguir el objetivo del rol. En segundo lugar, los comportamientos expresivos son sentimientos emocionales relacionados con el cumplimiento del propio rol. Una aproximación interaccional a los roles se describe como rol adquirido, esto es, uno aprende el rol interactuando con una persona en un rol complementario. Tal y como he descrito los roles se desarrollan en primarios, secundarios y terciarios. La base del modo de función de rol es la integración de los roles en el interior de la persona, así como las conductas colectivas de los roles complementarios.

La historia que cuento para ilustrar el modo de función de rol trata sobre una paciente que cuidé en una unidad de cuidados intensivos. Esta mujer estaba en coma, y me asignaron su cuidado. Estos fueron complicados debido a las numerosas necesidades fisiológicas, incluyendo los cuidados del respirador y de la traqueotomía, el balance de entradas y salidas a través de las vias intravenosas. Recibia múltiples medicaciones, y por esta época no respondía a estímulos. Mientras la cuidaba, me di cuenta que las enfermeras eran especialmente amables con los pacientes. Siempre hablaban a los pacientes mientras les administraban cuidados, aunque estuvieran en coma. Pero, me di cuenta que a esta mujer le llamaban por su nombre de pila. Estuve pensando sobre qué otros modos adaptativos están involucrados junto al modo fisiológico. Se me ocurrió que siendo una mujer mayor en un ambiente extraño y de acuerdo con su rol primario, sería más apropiado llamarla por su apellido. Lo hice mientras la cuidaba, y noté una débil respuesta por su parte. Esa tarde estuve en la ronda de neurología, a la que se le presentó esta paciente al personal médico. El neurólogo explicó cuidadosamente porque nunca se recobraria del coma irreversible. La mañana siguiente, mientras la cuidaba, la encontré un poco más reactiva. El final de la historia es que ocho días más tarde, ella se marchaba caminando del hospital. Cuento la historia no para decir que el Modelo de Adaptación de Roy saca a la gente del coma. Más bien lo hago para decir que uso el Modelo de Adaptación de Roy incluso cuando cuido personas en la UCl.

El modo adaptativo final es interdependencia. En este modo, las enfermeras se centran en las interacciones relacionadas con dar y recibir amor, respeto y ánimo. La necesidad subyacente para un grupo es el contexto social. El modo de interdependencia para un individuo tiene dos componentes, las personas significativas y los sistema de apoyo. Lo paralelo para el grupo son el contexto, infraestructura y recursos. El modo de interdependencia con sus sub-áreas y componentes se ilustra de la siguiente forma. Los estímulos incluyen fac- 
tores externos como los económicos, sociales, políticos, culturales, religiosos y sistemas familiares. Los estímulos internos serían la misión, la visión, valores principios, logros y planes. Esto proporciona el contexto del modo de interdependencia a nivel del grupo. Los niveles de adaptación para el modo de interdependencia incluyen las infraestructuras de los procesos relacionales, de desarrollo y de acceso a los recursos. Las personas son la clave en el modo de interdependencia tanto para los individuos como para los grupos. Cada persona tendrá habilidades de convivencia que incluirán conocimientos, destrezas, convencimientos y actitudes.

Para resumir primer gran concepto del modelo, los sistemas adaptativos humano, podemos poner juntos los mecanismos internos de convivencia actuando a través de cuatro modos para interiorizar, procesar, y responder al medio ambiente.

El concepto clave de medio ambiente se define como: todas las condiciones, circunstancias e influencias que rodean o afectan el desarrollo o el comportamiento tanto de personas como de grupos, con consideraciones particulares de la mutualidad entre personas y recursos naturales. El medio ambiente incluye la clasificación de estimulo que hemos encontrado útil en la práctica enfermera. Los estímulos focales son estímulos externos o internos que confrontan de inmediato el sistema humano. Los estímulos contextuales son todos los otros presentes en la situación que contribuyen al efecto de los estímulos focales. Finalmente, los estímulos residuales es un factor ambiental del interior o el exterior del sistema humano cuyos efectos sobre la situación actual no están claros. Estas clasificaciones están basadas en el reciente trabajo de un fisio-psicólogo, Helson.

El tercer gran concepto del metaparadigma de la enfermería es la salud. Usando el modelo de adaptación, discutiremos tanto sobre la salud como sobre la adaptación. La definición revisada de adaptación es: el proceso y el logro por el cual los pensamientos y sentimientos de las personas, como individuos o en grupo, son usados para percepción consciente y elección para crear una integración medio ambiental y humana. Al enterder la adaptación, describimos las respuestas adaptativas como aquellas que promueven la integridad en términos de logros del sistema humano. Estos logros incluyen la supervivencia, crecimiento, reproducción, adiestramiento e integración de la persona o del medio ambiente. Por otro lado, las respuesta inefectivas son aquellas que no contribuyen en el alcance de los logros del sistema humano.

Basado en el trabajo de Helson, el trabajo reciente sobre el modelo, hemos definido nivel de adaptación como el determinado por la mezcla de efectos de los estímulos focales, contextuales y residuales. Después, hemos continuado desarrollando la comprensión de los procesos básicos involucrados en la adaptación. Basado en este trabajo, creemos que podemos representar el nivel de adaptación en tres niveles diferentes, los procesos vitales que son los integrados, aquellos que son compensatorios y aquellos que están comprometidos. El proceso vital integrado es el nivel de adaptación en el cual las estructuras y funciones vitales como la respiración o desarrollo del auto-concepto están trabajando como un todo para cubrir las necesidades humanas. Los procesos compensatorios representan en nivel de adaptación en el cual los sistemas cognitivo y regulador han sido activados por una amenaza a los procesos vitales integrados. Un ejemplo de proceso compensatorio puede ser la hemoglobina que gradualmente se concentra cuanto mayor es la altitud donde vive la persona, pues necesitará más poder de transporte de oxígeno cuando el aire es menos oxigenado. Últimamente, el nivel de adaptación de los procesos comprometidos resultan de procesos vitales integrados y compensatorios inadecuados. Esto se conoce 
también como problemas de adaptación. Hemos marcado los problemas más frecuentes en cada uno de los modos adaptativos y los hemos comparado con la lista de los problemas de adaptación de la NANDA.

La adaptación conduce a la salud. De acuerdo con el modelo de adaptación, la comprensión del concepto de salud tiene raices en las premisas cientificas y filosóficas del modelo. El principio de Veracidad afirma que hay objetivos comunes a todos los seres humanos, y según mis creencias, creo que toda la vida creada y que los seres humanos comparten objetivos comunes. Además, desde la perspectiva de la unidad cósmica, uno se da cuenta de la compleja auto-organización de las personas y el medio ambiente. Incidiendo en el principio de veracidad, las premisas del modelo reconocen un significativo destino de convergencia del universo de las personas y el medio ambiente en un Dios-creador. Estos situaciones comunes de nuestro universo se ilustran con los siguientes ejemplos. La primera nos proporciona una exposición prolongada del cielo del norte, de la estrella polar. La segunda es una concha hemisférica. En segundo lugar vemos unos gráficos generados por ordenador de una vista axila de una molécula de ADN. Lo podemos comparar con una mancha de una ventana con cristal sucio, lo que ocurre al representar el primer libro del Génesis, la creación. Aquí vemos las similitudes de situación y forma en el universo de los seres vivos y en nuestro medio ambiente. Con el concepto de adaptación y las principales premisas en mente, defino la salud como un estado y un proceso de ser y llegar a estar integrado y completo.

Describiendo el modelo de enfermería, el último concepto clave es el de enfermería. De acuerdo con el modelo de Roy, la enfermería ve a las personas como co-extensiones con su ambiente físico y social. Más allá, la enfermería toma en consideración los valores, esperanzas y creencias de las personas como creadores de futuro. Un autor, un científico escolar el cual cito es T. de Chardin. En sus escritos estableció claramente que "todo el futuro de la tierra me parece que depende del reconocimiento de la fe en nuestro futuro". Así, veo la enfermería hoy muy orientada al futuro y promoviendo la salud a través de la promoción de la adaptación y procurando la integración total de la gente en el universo dirigido a un destino común.

Como definición de enfermería, uso dos. En general, defino enfermería como: el profesional del cuidado centrado en los procesos y situaciones humanas, y enfatiza en la promoción de la salud de las personas, las familias, grupos y la sociedad como conjunto. Para una definición de enfermería de acuerdo con el Modelo de Adaptación de Roy, especifico lo siguiente: la ciencia y la práctica que aumenta las habilidades adaptativas y procura transformaciones en las personas y en el medio ambiente.

\section{Ejemplos de aplicación en la práctica.}

Podemos ver algunos ejemplos de aplicación del Modelo de Adaptación de Roy en la práctica enfermera. La práctica clínica efectiva es debida tanto a la base de conocimientos como a la importancia social. Un modelo para la práctica,, debe proveernos del conocimiento para la práctica efectiva. El modelo también proporciona a la enfermera un rol, por el cual hace una importante contribución al bien social. Los modelos conceptuales son una vía para desarrollar conocimientos para la práctica efectiva y relevancia social. Así, el MAR es un ejemplo de modelo conceptual. Los modos adaptativos proporcionan la base del conocimiento científico para la práctica, así como para el juicio clínico. En el repaso del modelo, hemos visto que el sistema adaptativo humano está compuesto de procesos centrales adaptativos, 4 modos adaptativos, y que al mismo tiempo, recibe estímulos del medio ambiente, tanto internos como externos, y que tiene un sistema de respuestas en forma de comportamientos. 
Un ejemplo de cómo el conocimiento se desarrolla basando en la práctica con el modo fisiológico puede ser la necesidad de oxigenación. Al describir este modo, vemos un proceso vital básico, particularmente la ventilación, intercambio de gases transporte. Al describir el modelo ya hemos descrito las respuestas adaptativas de la oxigenación. Algunas de estas respuestas son innatas, como el reflejo de la los. Otras son aprendidas, como el entrenamiento de los músculos de la respiración. Si la persona tiene problemas para resolver sus problemas de oxigenación y los procesos compensatorios no son suficientes para mantener la integración, entonces la persona puede desarrollar respuestas adaptativas comprometidas. Dos ejemplos que ocurren con frecuencia en los procesos adaptativos comprometidos en esta necesidad son la hipoxia y shock.

Para proporcionarles un ejemplo específico, usaré un paciente que tenía serios problemas en la función neurológica del modo fisiológico. Como ya dije antes, el objetivo de la enfermería es la promoción de la adaptación. Y promoción de la adaptación incluye a la enfermería interviniendo aumentando las capacidades adaptativas y potenciadoras del medio ambiente. El ejemplo que les quiero contar es el de un joven adulto traído al servicio de urgencias por un accidente de tráfico. Aunque llegó inconsciente, recuperó la conciencia pero estaba desorientado. Conversaba y obedecía órdenes, pero no recordaba nada del accidente. Refería fuertes dolores de cabeza, molestias visuales, mareo, y nerviosismo. Según iba pasando el tiempo, el dolor de cabeza empeoraba, evolucionó hacia un cuadro de inestabilidad y confusión y finalmente tuvo una convulsión. Se diagnosticó hematoma subdural tras un TAC, y se inició una intervención quirúrgica. Los comportamientos que la enfermera registró se pueden resumir en presión arterial elevada, dolor de cabeza empeorándose, mareos, confusión, dificultad cognitiva y convul- siones. El estímulo más importante era en primer lugar, el accidente de tráfico, y el interno, el aumento de la presión intracraneal. El diagnóstico de enfermería aplicable en este caso podría ser descenso del nivel de conciencia debido a los signos de aumento de la presión intracraneal.

En esta situación al usar el MAR, el conjunto de objetivos incluye trabajar con la familia para establecer claramente los resultados de los cuidados de enfermería. Un objetivo completo incluye el comportamiento de nuestro centro de atención, el cambio esperado, y el tiempo en el que se espera que se complete el cambio. Los objetivos pueden ser a largo o a corto plazo, esto depende de la situación en cuestión. De todas maneras, al trabajar con personas con problemas del sistema nervioso, es característico que los objetivos tienden a ser extremos, siendo inmediatos, como en los cuidados críticas, o muy a largo plazo, como en la rehabilitación y el cuidado de las personas mayores.

Para el joven con el nivel de conciencia bajo por el aumento de la presión intracraneal, el objetivo general es evitar la aparición de lesiones cerebrales y complicaciones debidas al coma. Específicamente, la enfermera de noche estableció que en 30 minutos la causa del aumento de la presión intercraneal, sería identificada y se iniciaría la medida para aliviar la presión. Las acciones de enfermería deberían incluir las siguientes: apertura de vía aérea; ventilación y circulación adecuada; observar/registrar cambios neurológicos minimos; ambiente tranquilo; cabecera de la cama elevada 30 grados con la cabeza alineada con el cuerpo; prevenir súbitos aumentos de la presión; y minimizar el trauma emocional y físico.

Algunos valores y creencias clave desde las premisas del modelo de Roy que son importantes al aplicarlo en la práctica con grupos son: diversidad, común sentido y destino de la creación; habilidades humanas creativas capaces de transformar el universo; y encontrar sentido a las relaciones mutuas 
con el mundo creado y con el Dios-figura.. Para los grupos, los modos adaptativos incluyen: lo físico, la función de rol, y la interdependencia. Los procesos de convivencia interna son llamados el estabilizador y el regulador. Los estímulos son recibidos por el sistema y son procesados por los procesos de convivencia resultando el comportamiento.

El subsistema estabilizador es proceso de convivencia de grupo que está asociado con el sistema de mantenimiento y están incluidos: estructuras, valores y actividades diarias, por los que los participantes cumplen los propósitos del sistema social. Así como el subsistema regulado mantiene el balance interno de la persona, el sistema estabilizador mantiene el sistema entre los límites de la normalidad. El subsistema innovador es un grupo de procesos convivencia que incluyen estructuras y procesos para el cambio y el crecimiento. El grupo no puede mantenerse por sí mismo, pero respondiendo a los cambios del exterior debe cambiar también y crecer. Así pues, el subsistema se considera significativo para la adaptación del grupo.

En el modo físico, la necesidad del grupo es la integridad del sistema. Los componentes incluyen operaciones básicas con los recursos, como la familia consiguiendo comida o una casa; los participantes, como un paciente en una unidad de cuidados teniendo una enfermera para cuidarle; facilidades físicas, cómo un equipo de béisbol teniendo un lugar donde poder jugar; y recursos fiscales, esto es el dinero para comprar comida, hospedaje, pagar a las enfermeras, o comprar los uniformes de el equipo.

El modo de identidad del grupo refleja la necesidad del grupo de mantener la integridad de su identidad. Los componentes de este modo incluyen compartir relaciones, objetivos y valores. Un ejemplo de objetivo común podría ser el equipo de béisbol cuyos miembros quieren ganar el juego. En segundo lugar, un medio social y cultural son componentes importantes también por el cual el grupo pueda transmitir y dar a conocer sus valores a todos los miembros del grupo. La imagen que tiene el grupo de sí mismo incluiría cosas como el grupo viéndose así mismo como ganadores o perdedores. Finalmente, la co-responsabilidad de alcanzar el objetivo significaría que cada uno de los miembros debería hacerlo lo mejor posible con tal de alcanzar el objetivo común, esto es, jugar bien cuando están el campo, e intentar marcar cuando están bateando.

La necesidad básica del modo de función de rol es la integración social y clarificación del rol. Los componentes de este modo son comprender y mentalización para cumplir con las tareas asignadas. Cada miembro del grupo necesita saber que se espera de él, y deben estar mentalizados para cumplir con las expectativas. En segundo lugar, es importante para el modo de función de rol que el grupo vaya consiguiendo sus objetivos. Por ejemplo, un grupo de enfermeras está socialmente integrado y está clarificado su rol cuando las enfermeras están mentalizadas para proporcionar cuidados adecuados, y todas entenderán qué significa esto y cuales son las tareas específicas para proporcionarle un buen cuidado.

El modo de interdependencia tiene como base la necesidad de relaciones integradas en un contexto social. Los componentes de este modo son, primero, adecuación de los recursos, relaciones y desarrollos de las infraestructuras. Esto significa que la estructuras alrededor del grupo tienen que contribuir a proporcionar los recursos que el grupo necesita. Por ejemplo, en nuestro sistema de salud, las compañias de seguros deben pagar sus facturas a los hospitales para que estos continúen proporcionando cuidados a las personas. En segundo lugar, el modo de interdependencia incluye el conocimiento, habilidades, mentalización y actitudes de las personas. De esta manera, el grupo es dependiente de cada persona contribuyendo al conocimiento, habilidades, así como a las mentalidades y las actitudes comunes. 
Un ejemplo de conocimiento para una práctica eficaz puede ser el de la enfermería que se realiza en atención primaria con las familias. Es ejemplo en particular se ha tomado del libro de texto de la revisión del MAR y trata sobre una pareja de ancianos en su casa. El señor y la señora E. eran una pareja cuya edad estaba entre los 70 y los 80 años. Estaban sanos y vivían solos en su casa. De todas maneras, al pasar cinco años, el señor E. empezó con signos de deterioro de su capacidad mental. Su memoria fallaba, su capacidad de juicio diminuyó, y con frecuencia tenía dificultades para tomar decisiones. A veces se desorientaba y no reconocía a sus familiares. La sra. E. también describía movimientos repetitivos y cambios de personalidad. Ella decía "Siempre fue un marido cariñoso. Tenía una buena relación. No puedo entender qué le ha pasado". De hecho, a veces se ponía agresivo. Por ejemplo, intentando ayudar al sr. E. en su baño, se volvió hostil y le empujó. El sr. E. fue examinado por un médico hace un año y parecía que tenía algunos de los síntomas de la enfermedad de Alzheimer. En ese momento la sra. E. tomó la decisión de mantener a su marido con ella en casa todo el tiempo que fuera posible. Ahora, sin embargo, necesitaba ayuda. Ella, junto con la enfermera, estuvieron viendo qué se podía hacer para hacer posible que la pareja continuara viviendo en su casa. Cuando se preguntaron por las personas allegadas, la sra. E. dijo que además de a su marido, ella tenía un hijo y una hija, cuyas familias residían a media hora de tren desde su casa. Una vez a la semana su hija llevaba comida y algunas frutas. Los sábados un nieto llevaba a la sra. E. a comprar alimentos, mientras el nieto favorito del sr. E. le llevaba a pasear. Además, hasta hace poco formaban parte de un club social, y cuando el sr. E. empezó a empeorar, muchos de sus amigos iban a visitarle y a menudo le llevaban comida o flores. La sra. E. fue enfermera. Pensó que sería capaz de sacar adelante a su marido, pero ahora empezaba a sentir que no podía manejarle sola todo el tiempo, particularmente cuando no podía dormir durante la noche. Se preguntaba como ayudar a su marido si sus capacidades funcionales continuaban disminuyendo. La sra. también se preocupaba por la seguridad de su marido en su medio ambiente. Tenía que mantenerle encerrado en la casa la mayoría del tiempo, lo que incrementaba su enfado y su agitación. Recientemente la sra: E. le preguntó a la enfermera que si se podía hacer algo más para aumentar la seguridad en su medio y evitar problemas potenciales.

Para las aplicaciones clínicas, podemos centrarnos en tres diagnósticos específicos. El primero es relativo a la sra. E.: convencimiento para cuidar en casa a su marido con problemas mentales en relación con el cariño por él, confianza en sus posibilidades, y con la ayuda proporcionada por sus allegados. El segundo diagnóstico es para el sr. E. e incluye: aumento de la agitación, agresividad, y riesgo de accidente debido al medio que no le proporciona ni libertad ni seguridad. El tercero es relativo, de nuevo a la sra. E. Primero, el cansancio y extenuación debido a los problemas de sueño de su marido. En segundo lugar, cuestionarse sobre la capacidad de convivir con el descenso de las habilidades funcionales de su marido, debido a la falta de conocimiento sobre las aproximaciones y acciones efectivas de soporte a un individuo con problemas mentales. Sería importante para la enfermera para trabajar con la sra. E. el marcarse objetivos reales y desarrollar aproximaciones para conseguir sus objetivos.

\section{Dificultades al Aplicar el Modelo.}

Las dificultades al aplicar el modelo pueden ser identificadas en relación con la práctica enfermera y en relación con la educación de las mismas. En primer lugar, déjenme decirles que hace unos 15 años que al menos 100.000 enfermeras fueron educadas durante la carrera con el Modelo de Adaptación de Roy en los Estados Unidos y er: Canadá. Creo que el número puede ser tres veces 
mayor ahora, y ahora se podrían añadir quizás cientos de enfermeras educadas en otros países. Puede ser cierto que en algún momento toda enfermera haya tenido alguna dificultad al aplicar el Modelo de Adaptación de Roy, pero el balance de muchas enfermeras es que han encontrado las ventajas de una herramienta clara, más que desventajas.

Todavía una crítica común de las teorias es que están muy distantes de la realidad de la práctica. Algunas teóricas son criticadas por no estar en contacto con la práctica. En mi propio trabajo he tenido el privilegio de usar la práctica para ayudar al desarrollo del modelo. Por ejemplo, al tener estudiantes recogiendo datos de 500 pacientes que fueron utilizados para identificar los modos adaptativos, o las maneras de manifestarse sus niveles de adaptación. Mi vida parece conducirme a la práctica bastante a menudo, ya que cuando escribo sobre el modelo siempre pienso en la práctica. Aún siendo capaz de usar el modelo con eficacia en la práctica, depende del nivel de comprensión tanto del modelo como de la especialidad clínica. Por ejemplo, aparentemente no se identifica de inmediato cómo se ve a sí mismo un niño, esto es una dificultad. De todas maneras, he conocido enfermeras creativas que utilizan datos como ver si se llama al niño por su nombre o no, como un signo para hacer posible la valoración del desarrollo del auto-concepto del propio niño.

Otra dificultas encontradas por algunos es que los modelos de enfermería o las teorías usan un lenguaje poco usual en la práctica enfermera. El Modelo de Roy utiliza muchos términos que son comunes en la enfermería como los términos de los pasos de proceso de enfermería: reconocimiento, diagnóstico, objetivos, intervenciones y evaluación. Además muchas etiquetas de los problemas de adaptación, como hipoxia o pérdida, son familiares para las enfermeras en la práctica. De todas maneras, desarrollando el modelo hemos introducido algunos términos nuevos. La teoría de siste- mas puede ser un concepto bastante común, pero debido a los avances científicos del siglo XX en la exploración del espacio y en la cosmología se han añadido nuevos términos. Me gustaría pensar que terminología como "las personas y la tierra tienen ciclos comunes y relaciones integrales" ayuden a las enfermeras a identificarse con los cambios que se producen en el mundo a su alrededor. Las enfermeras se han centrado durante muchísimo tiempo en las personas su medio ambiente, pero esto puede tener un significado más profundo si uno se toma su tiempo para comprender las premisas científicas y filosóficas para el siglo XXI.

En la educación a las enfermeras, una dificultad es que en las diferentes facultades pueden tener distintos niveles de comprensión del modelo. Los estudiantes necesitan una guía consistente al mismo tiempo que les enseñen de forma creativa. Si alguna facultad no tiene una buena comprensión de las premisas y de los conceptos clave del modelo, la consistencia necesaria para aplicarlo entre una rotación clínica y otra puede llevar a los alumnos a tener algunas dificultades. Siempre que un grupo, como una facultad, decide probar nuevas ideas, la gente del grupo presentarán resistencias al cambio. El cambio siempre es difícil y si uno no está concienciado en el cambio se producen dificultades tanto para la persona como el proyecto del grupo. Estas dificultades no son exclusivas al aplicar un modelo, pero se dan en cualquier cambio. A pesar de todo, al introducir un modelo en la educación de las enfermeras, es particularmente importante tener suficientes personas que sean instruidas y convencidas de que van a conseguir la implantación del modelo. Otra posible fuente de dificultades puede ser la falta de apoyo administrativo. He conocido muchos proyectos de implementación que no han tenido éxito cuando se ha producido un cambio en la enfermera que lideraba el proyecto por otra que no lo apoyaba. Al hacer el cambio, las personas invierten muchas horas 
extras, energía y esfuerzo personal, no hay nada más desmoralizantes que el que no se reconozca el esfuerzo realizado. Si el líder tiene otros objetivos, los esfuerzos no serán tomados en cuenta, y probablemente el proyecto no tendrá éxito.

Una ventaja para cualquiera que aplique el Modelo de Adaptación de Roy en la práctica, o en la educación enfermera, es que muchos otros lo han hecho antes. Algunos han compartidos sus experiencias en la literatura enfermera. Los libros que hemos escrito sobre el modelo están basados en la experiencia de cientos de estudiantes en la escuela, que fue el primer lugar donde se implementó. Hay materiales para la docencia y la práctica disponibles en inglés, y también las hay traducidas a varios idiomas

\section{Conclusión.}

Para terminar, quisiera decir que ha sido un privilegio trabajar en el desarrollo de este modelo. conceptual para la práctica enfermera desde hace más de 30 años. Uno de los beneficios que he tenido como resultado de mi trabajo, ha sido tener la oportunidad de compartirlo con personas de diferentes partes del mundo. Espero que esta corta presentación, y la discusión que tendremos, les proporcione una mejor comprensión del Modelo de Adaptación de Roy, sus premisas, sus conceptos clave, su aplicación, y la aportación que pueden hacer a la enfermería y a la cultura de los cuidados que esto crea.

(Traducción realizada por David Molero Tolino)

\section{THE ROY ADAPTATION MODEL IN THE CONTEXT OF NURSING MODELS WITH EXAMPLES OF APPLICATION AND DIFFICULTIES}

It is a real privilege to be with you today at this important conference and to have the opportunity to share my work on the Roy Adaptation
Model (RAM). During this session, we will talk about nursing models in general. Secondly, I will describe the RAM specifically, including the philosophical and scientific assumptions as well as the key concepts. Thirdly, I will provide examples of applications of the model in practice and conclude with a discussion of some of the difficulties encountered when the model is used in practice and in nursing education.

\section{Nursing Models}

A model is simply a representation of reality. When we are building a new section of a health care center, we often place a model of it on a table at the entrance of the old hospital building. The model may be made of wood or plastic parts that represent the building project. For example it shows how a new out-patient clinic building will be connected to the parking lots and to other buildings already present. The model of the new construction represents what it will look like. It also shows us the parts and how they relate to one another. Nursing has found it useful to develop models to represent the reality of nursing, to show the various parts, and how they relate to one another. These models are constructed of concepts and figures that represent the parts of nursing. Another example of a model might be the artist's painting of a tree. We have all seen beautiful trees painted by van Gogh. There are also paintings by van Gogh that show blossoms on a tree close-up. A nursing model, then, provides us both with an overview and with the close-up view of the parts that represent our beliefs about nursing.

It is important to note that models of nursing are representations of the reality of nursing practice. What happens between patients and nurses and how nurses contribute to caring and to helping improve health is the subject matter for nursing models. As models for nursing practice began to be 
developed in the United States in the 1970s and the 1980s, it was noted that looking across different models, we could find the same basic elements. Models include a clear concept of the person. Understanding the human person is based on our philosophy as well as our scientific view of persons and their place in the world around. In addition, nursing models in describing the world that people interact with are describing what we call environment. The other two major concepts are the notion of health and the idea of nursing itself. Nursing models then are depictions of nursing practice that are expressed using the essential concepts of person, environment, health, and nursing.

\section{The Roy Adaptation Model}

As a model of nursing, the RAM has philosophical and scientific assumptions that relate specifically to the person and the world in which they interact. Scientific advances of the 20th century, particularly quantum theory and explorations of the universe, have opened the way for new visions of persons and environment in the new millennium. Based on these developments, I would like to share my more recent work on assumptions of the Roy model for the 21 st century.

Scientific assumptions for the 21 st century build upon the earlier systems assumptions and assumptions related to adaptation. However, they add ideas from understanding persons' place within the total universe. These assumptions are described as follows: 1) systems of matter and energy progress to higher levels of complex self organization; 2) consciousness and meaning are constitutive of person and environment integration; 3) awareness of self and environment is rooted in thinking and feeling; 4) human decisions are accountable for the integration of creative processes; 5) thinking and feeling mediate human action; 6) system inter-relationships include acceptance, protection, and fostering of inter-dependence; 7) persons and the earth have common patterns and integral relations; 8) persons and environment transformations are created in human consciousness; and 9) integration of human and environment meanings results in adaptation.

The two basic philosophical assumptions that I used as early as 1988 were: 1) humanism, that is, the broad movement in philosophy and psychology that recognizes the person and subjective dimensions of the human experience as central to knowing and valuing; 2) veritivity, that is, a principle of human nature that affirms a common purposefulness of human existence. Out of this and more recent experiences in a community of scholars with faith foundations, I began to develop philosophical assumptions for the 21 st century. These include 1) persons have mutual relationships with the world and with a God-figure; 2) human meaning is rooted in an omega point convergence of the universe; 3) God is intimately revealed in the diversity of creation and is the common destiny of creation; 4) persons use human creative abilities of awareness, enlightenment and faith; and 5) persons are accountable for the processes of deriving, sustaining and transforming the universe.

I'd like to summarize this section on the assumptions by simply noting that I have called this development cosmic unity. Cosmic unity refers to the mutual complex person and environment self organization, and secondly, the meaningful destiny of convergence of the universe, persons and environment in what I call a creator-God.

In describing the key concepts of the model, the first is seeing the person or group as an adaptive system with coping processes. The adaptive system is described as a whole comprised of parts. This system functions as a unity for some purpose. Finally, the human system includes people as individuals or in groups. This may be families, organizations, communities or society as a whole. Two key elements of understanding a person as an 
adaptive system are the adaptive processes and the adaptive modes. Adaptive processes include the activity of the subsystems for coping at the individual and group level. The adaptive modes are seen as ways of manifesting the adaptive processes. The central processes of adaptation are broadly described as the regulator subsystem and the cognator subsystem. The regulator subsystem includes all the neuro-chemical and endocrine channels whereby the person takes in the environment and makes almost automatic unconscious responses. Secondly, the cognator subsystem contains the perceptual information processing, learning, judgement, and emotion whereby again the internal and external stimuli are processes so that the person can make a response.

The story I often use to illustrate these two abstract concepts is that of the mother of a young child in front of their house. Imagine the child runs into the street when a car is coming. The mother runs and grabs the child out of the street to safety. In this case, her regulatory system has acted to give her all the adrenaline and the neurotransmitter signals that she needs to quickly make a response. At this point, the information does not even pass through the cerebral cortex. Rather, it travels through the spinal cord and allows her to act quickly. Secondly, after the child is safe in her arms, she goes through a process of thinking about the situation and also she recognizes her own emotion of anxiety and fear. She makes a new judgement and concludes that this child is so active she will need to put a fence around the yard. These two processes act together to help the person deal with changes in the environment both immediate and long-term.

The parallel internal processes of a group are the stabilizer subsystem and the innovator subsystem and these will be discussed later in an example for practice.

Four adaptive modes were identified in 500 samples of patient behavior. The first adaptive mode has been redefined as the physiologic and physical mode. The characteristics of this mode include the ways that humans interact as physical beings. This includes nine components for the individual with the underlying need for physiologic integrity. For the individual, the physiologic mode includes five basic needs: oxygenation, nutrition, elimination, activity and rest, and protection. In addition, the physiologic mode includes components that are based on the regulator subsystem, that is, the senses, fluid and electrolytes, neurologic function, and endocrine function. For groups, the physical mode involves manifesting adaptation relative to basic operating resources such as participants, physical facilities and fiscal resources.

The next adaptive mode relates to self-concept. This is who one is within oneself at any point in time. The woman in the figure is in a boat on Lake Titicaca in South America. The selfconcept/group identity mode is described as follows: the underlying need for the individual is psychic integrity, or the need to know who one is so that one can be or exist with a sense of unity. The underlying need for the group is identity-integrity. The components of the self-concept mode for the individual include the physical self, which is manifested in body sensation and body image. Secondly, the personal self includes self-consistency self-ideal, and the moral-ethical-spiritual self. The group identity mode concepts include interpersonal relationships, group self image, the social milieu and culture. The figure shows the group identity mode with its sub-areas and components. The group identity stems from shared relationships, goals, and values. In turn, this shared vision rests upon a group self image and co-responsibility for goal achievement. These exist within the general background of the social milieu and social culture.

Also, fom the large sample of patient behaviors, we identified patient needs related to what 
has been called the role function mode. In this photograph, we see women in many roles, mother, friend, and buyers and sellers in an open air market. The underlying need of the role function mode for individuals is social integrity; the need to know who one is in relation to others so that one can act. For any group, the underlying need of the role function mode is role clarity. We have found it useful in assessing roles to describe the role of any given person as a role set. The role set includes primary roles that are based on one's developmental stage, for example, a twenty-seven year old young adult female. This person may have several secondary roles that are related to the primary role, such as wife, mother, and teacher. Finally, tertiary roles include those that are less central to the person and maybe only temporary. For example, fashion consultant, cooks three evenings a week, baseball fan, university student part-time, association member, and football coach.

In updating the literature on the role function mode, we have added some terminology related to role sets being the individual roles of the person and the aggregate role set being the roles held by several different people at the same time. Furthermore, our understanding of role function mode includes the structural approach. This describes instrumental behaviors, that is, behaviors used to reach the goal of the role. Secondly, expressive behaviors are emotional feelings related to fulfilling one's role. An interactional approach to roles is described as role taking, that is, one learns a role by interacting with a person in a complimentary role. As l've described, roles develop as primary, secondary, and tertiary roles. The base of the role function mode is the integrating of roles within the person as well as the collective patterns of complimentary roles.

The story I tell to illustrate the role function mode involves a patient that I took care of in an intensive care unit. This woman was in a coma and
I was assigned to give her care. This care was complicated by many physiologic needs, including care of the ventilator and tracheotomy, the measuring of intake and output through intravenous lines and catheters. She was receiving multiple medications and was at that time non-responsive. As I took care of her, I was aware that the nurses in the unit were particularly kind to patients. They always spoke to them while they cared for them, even if they were in a coma. However, I noted that they called this woman by her first name. I was trying to think about what other adaptive modes were involved besides the physiologic mode. It occurred to me that she was an older woman in a strange environment and according to her primary role, it might be more appropriate to call her by her last name. I did this while I was taking care of her, and I felt a flicker of response from her. That evening I went to neurologic rounds, at which this particular patient was presented to the medical staff. The neurologist carefully explained why she would never recover from irreversible coma. The next morning while I was taking care of the patient, I found her a little more responsive. The end of the story is that eight days later, she walked out of the hospital. I tell the story not to imply that the Roy Adaptation Model brings people out of a coma. Rather, my point is that I use the Roy Adaptation Model when I give nursing care even in intensive care units.

The final adaptive mode is interdependence. In this mode, the nurse focuses on interactions related to giving and receiving of love, respect and value. The underlying need for the individual is relational integrity and the feeling of security in relationships. The underlying need for a group is social context. The interdependence mode for the individual has two components, significant others and support systems. The parallel components for the group are context, infrastructure, and resources. The interdependence mode with its sub-areas and components are illustrated as follows. The sti- 
muli include external factors such as economic, social, political, cultural, religious, and family systems. Internal stimuli might be mission, vision, values, principles, goals and plans. These provide the context of the interdependence mode at the group level. Adaptation levels for the interdependence mode include the infrastructure of relational processes, developmental processes and resource processes. People are the key to the interdependence mode for both individuals and groups. Each person will have coping abilities that include knowledge, skills, commitments, and attitudes.

To summarize the first major concept of the model, human adaptive systems, we can put together the internal coping mechanisms acting through the four modes to take in, process, and respond to the environment.

The key concept of environment is defined as: all conditions, circumstances and influences surrounding and affecting the development and behavior of both persons and groups, with particular consideration of the mutuality of person and earth resources. The environment includes the classification of stimuli that we have found useful in nursing practice. The focal stimuli are the internal or external stimulus most immediately confronting the human system. Contextual stimuli are all the other stimuli present in the situation that contribute to the effect of the focal stimulus. Finally, the residual stimulus is an environmental factor within or outside the human system whose affects in the current situation are unclear. These classifications are based on earlier work by a physiological psychologist, Helson.

The third major concept of the nursing metaparadigm is health. In using the adaptation model, we discuss both adaptation and health together. The revised definition of adaptation is: the process and outcome whereby thinking and feeling persons as individuals and in groups use conscious awareness and choice to create human and envi- ronmental integration. In understanding adaptation, we describe adaptive responses as those that promote integrity in terms of the goals of the human system. These goals include survival, growth, reproduction, mastery, and person and environment integration. On the other hand, ineffective responses are those that do not contribute to the integrity of the goals of the human system.

Based on the work of Helson, in the early work in the model, we had defined adaptation level as determined by the pooled effect of focal, contextual, and residual stimuli. Later, we have continued to develop understanding of the basic processes involved in adaptation. Based on this work, we thought it might be useful to represent adaptation level at three different levels - the life processes that are integrated, those that are compensatory, and those that are compromised. The integrated life process is the adaptation level at which the structures and functions of a life process such as oxygenation or development of self concept, are working as a whole to meet human needs. The compensatory processes represents the adaptation level at which the cognator and regulator have been activated by a challenge to the integrated life processes. An example of a compensatory process might be the hemoglobin of the blood gradually concentrating when a person lives at a higher altitude so that there will be more oxygen carrying power when the air is less dense with oxygen. Lastly, the adaptation level of compromised processes results from inadequate integrated and compensatory life processes.

These are also known as the adaptation problems. We have outlined the commonly occurring problems in each of the adaptive modes and compared them to the North American Nursing Diagnoses Association list of adaptation problems.

Successful adaptation leads to health. According to the adaptation model, an understanding of the concept of health is rooted in the scien- 
tific and philosophical assumptions of the model. Veritivity affirms a common purposefulness of human existence and in my belief system, I hold that there is a meaning and purpose to all created life and that human beings share a common purposefulness. Also, from the perspective of cosmic unity, one is aware of the mutual complex person and environment self organization. Expanding on veritivity, the model's assumption acknowledges a meaningful destiny of the convergence of the universe of persons and environment in a creator God. This common pattern of our universe is illustrated in the following images. The first one provides a timed exposure of the North Sky about the Pole Star. The second is a sundial seashell. Secondly, we have a computer graphics-generated axial view of a DNA molecule. We can compare this with a rose stainedglass window, which happens to represent the first book of Genesis, that is, creation. We see here the commonalities of pattern and form in the living universe and in our environment. Keeping in mind the concept of adaptation and the broad philosophical assumptions, I define health as a state and a process of being and becoming integrated and whole.

In describing the nursing model, the last of the four key concepts is the concept of nursing. According to the Roy model, nursing sees persons as co-extensive with their physical and social environment. Furthermore, nursing takes a valuesbased stance and believes and hopes in human persons as creators of the future. One author, a scientist scholar whom I quote is de Chardin. In his writings, he states most clearly that "the whole future of the earth seems to me to depend on the awakening of the faith in our future." Thus, I see nursing today as being very future oriented and promoting health through promoting adaptation and enhancing the total integration of people in the universe headed for a common destiny.

As for the definition of nursing, I have used two. In a general way, I define nursing as: a health care professional that focuses on human life processes and patterns, and emphasizes promotion of health for individuals, families, groups, and society as a whole. For a definition of nursing according to the Roy Adaptation Model, I specify as follows: the science and practice that expands adaptive abilities and enhances person and environment transformation.

\section{Examples of Applications in Practice}

We can now provide examples of application of the Roy Model in nursing practice. Effective clinical practice is both knowledge-based and socially accountable. A model for practice, then, must provide the knowledge for effective practice. The model also provides the nurse with a role whereby he or she makes important contributions to the social good. Conceptual models are one way to develop knowledge for effective practice and social accountability. The RAM is an example of a conceptual model that provides knowledge and social accountability. The adaptive modes provide the basis for the scientific knowledge for practice as well as clinical assessment. In the overview of the model, we saw how the human adaptive system is made up of central adaptive processes, four adaptive modes, and at the same time, receives input from the environment, both internal and external, and has system output in the form of behavior,

An example of how knowledge is developed for practice based on the physiologic mode can be provided from the basic need for oxygenation. In describing this mode, we look at basic life processes, particularly ventilation, gas exchange, and transport of gases, As we have further developed the model, we have described the compensatory adaptive responses in oxygenation. Some compensatory responses are innate, for example, the cough reflex. Other compensatory adaptive responses are learned, for example, respiratory muscle training. If the person is challenged in meeting his or her own oxygenation needs and the compensatory 
processes are not enough to maintain integration, then the person may have compromised adaptive responses. Two examples of frequently occurring compromised processes in this adaptive mode need are hypoxia and shock.

To provide a specific example, I will use a patient who has major problems of neurologic function of the physiologic mode. As noted earlier, the goal of nursing has been defined as to promote adaptation. Secondly, promoting adaptation involves the nurse intervening to expand adaptive capacities and enhance the environment. The example I want to talk about is that of a young adult male brought into an emergency unit following a motor vehicle accident. Although he arrived unconscious, he regained consciousness but was disoriented. He was conversing and following commands, but could not recall anything about the accident. He described severe headache, visual disturbances, dizziness, and a sense of nervousness. As the time progressed, his headache worsened, he developed drowsiness and confusion, difficulty in thinking, and he ultimately had a seizure. A medical diagnosis of subdural hematoma was made following a CT scan and surgical intervention was initiated. The behaviors that the nurse notes could be summarized as increased blood pressure, headache worsening, drowsiness and confusion, difficulty thinking, and seizures. The most important stimuli are first, the motor vehicle accident, and them, internally, the increased intercranial pressure. The nursing diagnosis applicable in this situation might be decreased level of consciousness due to signs of increased intercranial pressure.

In this situation in using the RAM, goal setting involves working with the patient and the family to establish clear outcomes for nursing care. A complete goal statement includes the behavior of focus, the change expected and the time-frame in which the change is to be achieved. Goals may be either long-term or short-term and these time-frames are relative to the situation involved. However, when working with patients with disorders of the nervous systems, characteristically the goals tend to take the extremes of being very immediate as in critical care, or very long-term as in rehabilitation and care of the elderly.

For the young man with decreased level of consciousness due to increased intercranial pressure, the general goal is to prevent secondary brain injury and complications of coma. Specifically, the nurse night set the goal that within 30 minutes, the cause of increased intercranial pressure will be identified and steps initiated to alleviate the pressure. Nursing interventions would include the following: open airway; adequate ventilation and circulation; observe/report slight neurological change; quiet environment; elevate head of the bed 30 degrees with the head in alignment with the body; prevent sudden increases in pressure; and minimize emotional and physical trauma.

Some of the key values and beliefs from the assumptions of the Roy model that are important in applying the model in practice with groups include: diversity, common meaning, and destiny of creation; human creative abilities accountable for transforming the universe; and persons find meaning in mutual relations with the created world and a God-figure. For groups, the adaptive modes include: the physical, group identity, role function, and interdependence. The internal coping processes are called the stabilizer and the regulator. Stimuli are received by the system and processed by the coping processes with resulting behavior.

The stabilizer subsystem is a group coping process that is associated with system maintenance and it includes: structure, values, and daily activities, whereby participants accomplish the purpose of the social system. Just as the regulator subsystem maintains the internal balance of the person, 
so the stabilizer maintains the system within normal limits. The innovator subsystem is an internal group coping process that involves structures and processes for change and growth. The group cannot simply maintain itself, but in responding to changes external to itself must also change and grow. Thus, the innovator subsystem is postulated as significant for group adaptation.

In the physical mode, the need of the group is system integrity. The components involve basic operating resources, such as the family having food and a house; the participants, such as a patient care unit having nurses to staff it; physical facilities, such as a baseball team having a field to play on; and fiscal resources, that is, the funds to buy food, housing, pay the nurses, or buy uniforms for the team.

The group identity mode reflects the need for group identity integrity. The components of this mode include shared relations, goals and values. An example of a shared goal would be the baseball team whose members want to win the game. Secondly, a social milieu and culture are also important components in that the group has a way of letting its goals and values be known to all the members of the group. Group self-image would include such things as the group seeing themselves as winners or losers. Finally, co-responsibility for goal attainment would mean that each member would do their best to contribute to the goal, that is, playing well when they are on the field and striving to score runs when their team is at bat.

The basic need of the role function mode is social integrity and role clarity. The components of the mode are, firsts, to understand and have commitment to fulfill expected tasks. Each member of the group needs to know what is expected of them and they must be committed to carry out those expectations. Secondly, it is important to the role function mode that the group be achieving common goals. For example, a nursing care group has social integrity and role clarity when the nurses are committed to good patient care and they all understand what this means and what their particular tasks are in relation to achieving good patient care.

The interdependence mode has as its base the need for relational integrity and a social context. The components of this mode involve first, relational, developmental, and resource adequacy of the infrastructure. This means that the structures around the group have to be contributing to the resources that the group needs. For example, in our health care system, the insurance companies have to pay the bills so that the hospital can continue to give care to patients. Secondly, the interdependence mode involves the knowledge, skills, commitments and attitudes of people. In this way, the group is dependent upon each person contributing knowledge, skills, as well as commitments and common attitudes.

An example of knowledge for effective practice can come from nursing care in public health with families. This particular example is taken from the revision of the RAM textbook and involves an elderly couple at home. Mr. and Mrs. E. are a couple in their mid-seventies. They are both physically healthy and can continue to live in their own residence. However, over the past five years, Mr. E. has demonstrated a progressive deterioration in mental capacity. His memory is failing, he has decreased judgement, and often he has difficulty making decisions. At times he is disoriented and does not recognize familiar people. Mrs. E. also describes mood swings and changes in his personality. She stated, "He was always such a loving husband. We had a very good relationship. I can't understand what gets into him." In fact, at times, he becomes aggressive. For example, when their son was attempting to help Mr. E. with his bath, he became hostile and struck him. Mr. E. was examined by his physician over a year ago, and it 
was suggested that he had many of the symptoms of Alzheimer's disease. At that time, Mrs. E. determined to keep her husband with her at home as long as possible. However, now she needs help. She is working with the nurse practitioner to determine what can be done to enable the couple to continue residing at home. When asked about her significant others, Mrs. E. identified that in addition to her husband, she has a son and a daughter, whose families live within a half-hour train ride from their home. Once a week their daughter brings a meal and some fruit. On Saturdays, the grandchildren take Mrs. E. for groceries while Mr. E.'s favorite grandson takes him for a walk. Mr. and Mrs. E. had been very involved with a social club until recently, when Mr. E.'s condition worsened. Many of their friends continued to stop by for a visit, often bringing food items or flowers. During her career, Mrs. E. had been a nurse. She was confident in her ability to deal with her husband, but is now beginning to feel that she cannot handle him all the time, particularly when he does not sleep through the night. She has concerns about how to support him, as his functional abilities continue to decrease. Mrs. E. is also voicing concerns about her husband's safety in the environment. She has to keep him locked in the house most of the time and this increases his restlessness and agitation. Mrs. E. recently asked the nurse if there was more that could be done to enhance the security of the environment to prevent potential problems.

For clinical applications, we can focus on three specific diagnoses. Diagnosis 1 is related to Mrs. E.: commitment to at-home care for mentally impaired husband related to deep affection for him, confidence in own capabilities, and assistance provided by significant others. Diagnosis 2 is for Mr. E. and includes: increased agitation, restlessness and risk of mishap due to environment that does not provide freedom or security. The third diagnosis is again related to Mrs. E. First, the fati- gue and exhaustion due to sleep disturbances of her husband. Secondly, concern about ability to cope with decreasing functional abilities related to lack of knowledge about effective approaches and actions to support a mentally impaired individual. It would be important for the nurse to work with Mrs. E. to set realistic goals and to develop approaches to reach these goals.

\section{Difficulties in Application of the Model}

Difficulties in application of the model can be identified in relation to both nursing practice and nursing education. First, let me say that about 15 years ago we estimated that at least 100,000 nurses had been educated in the curricula using the Roy Adaptation Model in the United States and Canada. I believe that the number could be three times that many by now and be added to by perhaps thousands of nurses from many other countries. It may be true to say that at some time every nurse may have some difficulty in application of the RAM, yet on balance most nurses have found that the advantages in having a clear framework outweigh the disadvantages.

Still, a common criticism of the theories for practice is that they are too distant from practice. Sometimes theorists are criticized for not being in touch with practice. In my own work, I have had the privilege of using practice to help with the development of the model. For example, in having students collect the data from 500 patients that was used to identify the adaptive modes, or ways people manifest their levels of adaptation. My life seems to take me back to practice often enough that I always am thinking about practice when I am writing about the model. Still, being able to use the model effectively in practice depends upon one's level of understanding of both the model and the clinical specialty. For example, it is not immediately apparent how to assess self-concept in an infant and this presents a difficulty. However, I 
have known creative nurses who use data such as whether or not the baby was named at birth as an assessment to make as a possible indication of the infant's developing self concept.

Another difficulty that has been identified by some is that nursing models or theories use language that is unfamiliar and not common in nursing practice. The Roy Model uses many terms that are common in nursing, such as the terms of the steps of the nursing process--assessment, diagnosis, goal setting, interventions, and evaluation. In addition, many labels for the adaptation problems, such as hypoxia or loss, are familiar to nurses in practice. However, in developing the model, we have also introduced some new terms. Systems theory may be a common enough concept, but adding the scientific developments of the 20th century in space exploration and cosmology add new terms. I would like to think that terminology such as "persons and earth have common patterns and integral relations" help nurses to identify with the changes in the world around them. Nurses have long focused on person and environment, but this can have a deeper meaning if one takes the time to understand the scientific and philosophical assumptions for the 21 st century.

In nursing education, one difficulty faced is that different faculty may have different levels of understanding of the model. Students need consistent guidance at the same time that they are taught to be creative thinkers. If some faculty do not have a sound understanding of the assumptions and key concepts of the model, the consistency needed for applications from one clinical rotation to the next may lead to difficulties for the students. Whenever a group, such as faculty, decides to try new ideas, people within that group will vary in their commitment to the change. Change is always difficult and when one is not committed to the change this presents difficulties for that person and for the group project as well. These difficulties are not uni- que to applying nursing models, but happen with any kind of change. However, in introducing a nursing model in nursing education, it is particularly important to have enough people who are both knowledgeable and committed to ensure successful implementation. Another possible source of difficulty may be the lack of administrative support. I have known several implementation projects that did not succeed when there was a change in the nurse leader from one who was very supportive to one who was not. In making change, people invest a lot of extra time, energy, and personal commitment and nothing is more demoralizing that not having this effort recognized as important. If, however, the leader has other goals, these efforts will not be viewed as important and likely the project will not succeed.

An advantage of to anyone applying the Roy Adaptation Model in practice or nursing education is the fact that many others have done this before. Some of shared their experiences in the nursing literature. The books that we have written on the model are based on the experiences of thousands of students at the school where the model was first implemented. Teaching and practice materials are available in English, and translations have been made into dozens of languages.

\section{Conclusion}

In closing, I would say that it has been my privilege to work on the development of this conceptual model for nursing practice for more than 30 years. One of the benefits I have received as a result of my work has been the opportunity to share the work with people in different parts of the world. I hope that this short presentation, and the discussion that we will have, gives you a better understanding of the Roy Adaptation Model, its assumptions, key concepts, and application, and the contribution that it can make to nursing and the culture of care that it creates. 